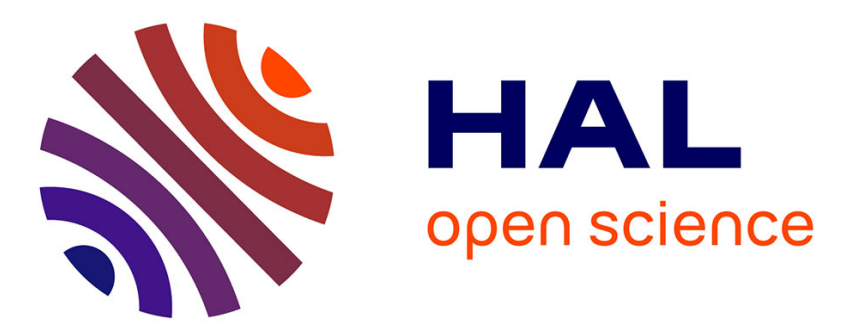

\title{
Importance of deformation-induced local orientation distributions for nucleation of recrystallisation
}

\author{
Romain Quey, Guo-Hua Fan, Yubin Zhang, Dorte Juul Jensen
}

\section{To cite this version:}

Romain Quey, Guo-Hua Fan, Yubin Zhang, Dorte Juul Jensen. Importance of deformation-induced local orientation distributions for nucleation of recrystallisation. Acta Materialia, 2021, 210, pp.116808. 10.1016/j.actamat.2021.116808 . hal-03387932

\section{HAL Id: hal-03387932 \\ https://hal.science/hal-03387932}

Submitted on 20 Oct 2021

HAL is a multi-disciplinary open access archive for the deposit and dissemination of scientific research documents, whether they are published or not. The documents may come from teaching and research institutions in France or abroad, or from public or private research centers.
L'archive ouverte pluridisciplinaire HAL, est destinée au dépôt et à la diffusion de documents scientifiques de niveau recherche, publiés ou non, émanant des établissements d'enseignement et de recherche français ou étrangers, des laboratoires publics ou privés. 


\title{
Importance of deformation-induced local orientation distributions for nucleation of recrystallisation
}

\author{
Romain Quey ${ }^{\mathrm{a}, *}$, Guo-Hua Fan ${ }^{\mathrm{b}}$, Yubin Zhang ${ }^{\mathrm{b}}$, Dorte Juul Jensen ${ }^{\mathrm{b}}$ \\ a Mines Saint-Etienne, Univ Lyon, CNRS, UMR 5307 LGF, Centre SMS, Saint-Etienne F - 42023, France \\ ${ }^{\mathrm{b}}$ Department of Mechanical Engineering, Technical University of Denmark, 2800 Kgs. Lyngby, Denmark
}

\section{A R T I C L E I N F O}

\section{Article history:}

Received 23 December 2020

Revised 8 March 2021

Accepted 10 March 2021

Available online 16 March 2021

\begin{abstract}
A B S T R A C T
Nucleation in an aluminium tricrystal cold rolled along its columnar direction, to 40\% thickness reduction, is studied. This experimental configuration was used to obtain the same deformation microstructure through the sample length, which made it possible to cut it into several slices of similar microstructures and use these slices differently. Some slices served to analyse the deformation microstructure and others to analyse the annealing microstructure. Nucleation developed only in one of the three crystals and not at grain boundaries. The relationship between the crystallographic orientations and the local density of nuclei and different attributes of the parent, deformation microstructure was then analysed. As generally presumed, the nuclei were observed to inherit orientations from the parent matrix. Much more surprisingly, the stored energy alone, which is often considered as the driving force for recrystallisation nucleation, was found not to provide a reliable criterion for recrystallisation nucleation in the investigated sample. Instead, the density of nuclei was the highest where the substructure is composed of sharp bands, which correspond to regions of highly anisotropic orientation distributions. A new energy criterion for recrystallisation nucleation is proposed, which is called "primary stored energy" and depends on the stored energy and the anisotropy of the orientation distribution.
\end{abstract}

(C) 2021 Published by Elsevier Ltd on behalf of Acta Materialia Inc.

\section{Introduction}

Recrystallisation nucleation in deformed polycrystalline materials is very challenging to study experimentally, as the nuclei are few and their locations difficult to predict. Even though it is well accepted that second phase particles, shear bands, transition bands, original grain boundaries and triple junctions are preferential nucleation sites, it is not known if they are equally favorable [1]. Moreover, relating the crystal orientation of a nucleus to that of its parent site, in the deformed polycrystalline matrix, is even more challenging, because as a nucleus forms, its parent matrix vanishes - a situation known as the "lost evidence problem" [2]. In principle, the lost evidence problem can be solved using threedimensional techniques such as three-dimensional X-ray diffraction microscopy [3] or white-beam differential-aperture X-ray microscopy [4], but a challenge remains to successfully select a region of the sample where nuclei will form (to be mapped before annealing) [5]. To date, this challenge has only been overcome by artificially stimulating recrystallisation nucleation using microhardness indentations [6] or focusing on orientation relations only [7].

\footnotetext{
* Corresponding author.

E-mail address: romain.quey@mines-stetienne.fr (R. Quey).
}

Another approach to study recrystallisation nucleation is to use microstructures such as carefully-deformed single crystals and bicrystals [8], or columnar polycrystals [9]. Columnar polycrystals, in particular, combine several advantages for recrystallisation studies: the grain orientations and shapes are relatively well known, the grain orientations can to some extent be selected to provide interesting orientation relationships, and the sample can be centimeter-sized, which yields a sufficient amount of material to pursue investigations under different annealing conditions. Even more importantly, as the sample microstructure does not vary significantly along the columnar direction, it becomes possible to cut the sample into several slices along this direction, consider them similar (even after deformation), and use and analyse them differently.

In this work, we analyse recrystallisation nucleation in a columnar tricrystal subjected to rolling. We relate the details of recrystallisation nucleation to the attributes of the parent, deformation microstructure at several scales, including the well-known "stored energy" associated to the dislocation boundaries (computed from the local disorientations [10]), as well as the local orientation distribution, and we propose a new energy criterion that describes our nucleation observations better than the stored energy alone. 
(a)

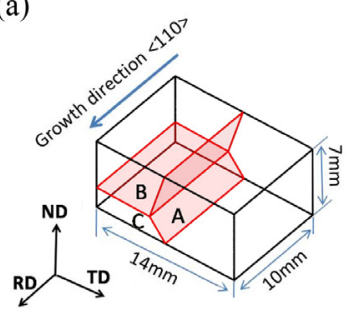

Grain boundary (GB)

(c)

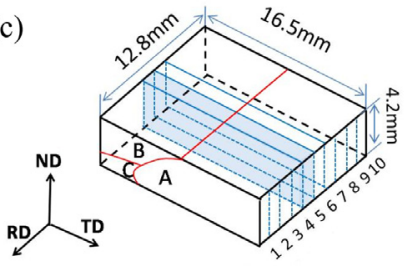

(b) $\{111\}$

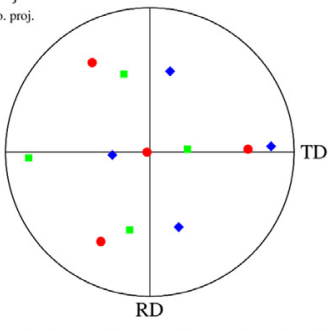

- Crystal A = Crystal B - Crystal C

(d)

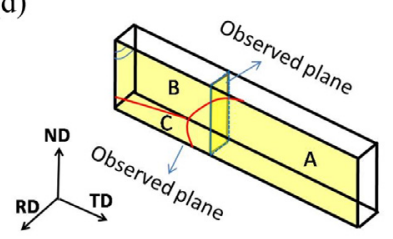

Fig. 1. Tricrystal aluminium sample and its partition into 10 slices. (a) Initial columnar microstructure with RD $\|\langle 110\rangle$, and crystals A, B and C, (b) orientations of the three crystals, (c) rolled sample and its 10 slices (after removing about $1 \mathrm{~mm}$ of material from both RD ends) with slice 5 highlighted in blue, and (d) observation planes of the slices.

\section{Experiment}

The initial material was a $\langle 110\rangle$-columnar tricrystal made of high-purity (99.99\%) aluminium and produced by directional solidification. A sample of $10 \mathrm{~mm} \times 14 \mathrm{~mm} \times 7 \mathrm{~mm}$ (along the rolling direction (RD), transverse direction (TD) and normal direction (ND), respectively) was cut so that the rolling direction was parallel to the columnar direction, RD $\|\langle 110\rangle$ (see Fig. 1a). The three crystals of the sample are denoted as A, B and C, and have orientations

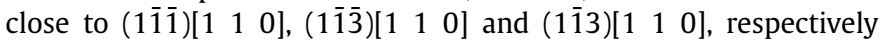
(see Fig. 1b). The three orientations are nearly self-symmetrical with respect to the TD-ND plane, and the orientations of crystals $B$ and $C$ are nearly symmetrical to each other with respect to the RD-ND plane. Moreover, and for future reference, these orientations are "moderately hard", "soft" and "soft" in terms of their Taylor factors for plane strain compression, which are about 3.7, 2.4 and 2.4 , respectively.

The tricrystal was cold rolled to $40 \%$ thickness reduction. Rolling was conducted in several steps, under the rolling geometry conditions of $l / h \simeq 2$, where $l$ is the contact length between a roll and the sample and $h$ is the mean sample thickness. As a result of the rolling, the tricrystal underwent the imposed normal (logarithmic) deformation of $\varepsilon_{33}=-0.51$ but also a transverse deformation of $\varepsilon_{22}=0.16$ corresponding to the widening of the sample typically occurring during rolling (while volume conservation yields $\varepsilon_{11}=0.35$ ). The sample was then cut into 10 slices along RD (see Fig. 1c). One slice was dedicated to characterizing the deformation microstructure (slice 5), while another one was used for analysing recrystallisation (slice 3 ). As expected from the specific experimental configuration, the two slices, which are close to each other and well away from the sample ends, exhibited similar microstructures after deformation, as will be seen from the deformation microstructure of slice 3 and the non-recrystallized part of the annealing microstructure of slice 5 (Figs. 3 and 6). Slice 3 was then annealed at $300{ }^{\circ} \mathrm{C}$ for $20 \mathrm{~min}$. In contrast to a previous work on the same sample [11], for which a slice was ground, before annealing, to artificially stimulate nucleation on its surface, in this work, slice 3 was electropolished to avoid this phenomenon.

The microstructures were observed using a Zeiss Supra 35 thermal field emission gun scanning electron microscope (SEM)

equipped with an HKL electron backscattered diffraction (EBSD) system. The characterization included electron channeling contrast (ECC), on full sample sections, and EBSD, on local areas, on the ND-TD and ND-RD planes (see Fig. 1d). For the EBSD maps, step sizes of 2 and $0.25 \mu \mathrm{m}$ were used for standard and high-spatialresolution observations, respectively.

\section{Deformation microstructure}

The deformation microstructure is analysed in the central slice of the sample (slice 5). Focus is on a large region about the triple line. Triple lines are well-accepted as preferential recrystallisation nucleation sites [1]. It is generally where crystals interact strongly with each other and where intense deformation heterogeneities can occur [12].

\subsection{Orientation distributions}

An EBSD orientation map of an area close to the triple point, in the TD-ND plane, is provided in Fig. 2a, and the disorientation ${ }^{1}$ profiles along the lines plotted in Fig. 2a, inside the three crystals, are shown in Fig. 2b. Disorientation profiles are provided both with respect to the initial pixel and between neighbouring pixels. Crystal A exhibits appreciable orientation changes at the micrometer scale, corresponding to fine microbands, that combine with a continuous orientation change at the millimeter scale, corresponding to a deformation gradient. Crystals $B$ and $C$ mainly show relatively small local orientation changes, with only a few large orientation changes that coincide with the edges of 100 to $200-\mu \mathrm{m}$ thick diffuse transition bands. The orientation distributions of the three crystals are represented as $\{111\}$ pole figures in Fig. 2c. Crystal B and, to a lesser extent, crystal C appear to develop crystal rotations towards distinctly different orientations, as apparent from their multimodal orientation distributions (this is also the case for crystal $A$, but in a region different to the one shown in Fig. 2a, as will be illustrated in Fig. 3). This phenomenon was already observed in a previous work [13], for specific grains of a sample deformed in plane strain compression, and was termed "orientation fragmentation". These grains were found to have symmetrical orientations (with respect to the sample axes), and their fragmentation behaviour was explained from the stability properties of the reorientation velocity field around them [13]. The orientation fragmentation observed in the three crystals of the present work (see Fig. 2c), which also are symmetrical orientations, can be fully explained from the arguments provided in this previous work [13] and from additional, micromechanical arguments provided in a related work [14]. First, because of the (self-)symmetry of the orientations of the three crystals with respect to the TD-ND plane, the orientations tend to change only slowly on average during deformation, by retaining their symmetry condition. Second, as the orientations show unstable reorientation conditions about their RD symmetry plane (i.e., orientations slightly away from the symmetry condition would rotate further away from it as deformation proceeds [13]), they tend to broaden by rotations about directions perpendicular to RD. Last, independently of their orientations, deformed crystals tend to develop larger slip variations on their most active slip systems [14]. During rolling, the most active slip systems have lattice spin vectors nearly parallel to TD, and so orientation fragmentation preferentially occurs by rotations about TD [14]. In this experiment, this is particularly clear for crystal B (see Fig. 2c).

An EBSD orientation map of an area close to the triple point, in the RD-ND plane, is shown in Fig. 3a (see Fig. 1d for the location

\footnotetext{
${ }^{1}$ In the article, "disorientation" refers to the rotation (or orientation difference) of minimal angle between two orientations under the conditions of crystal symmetry.
} 


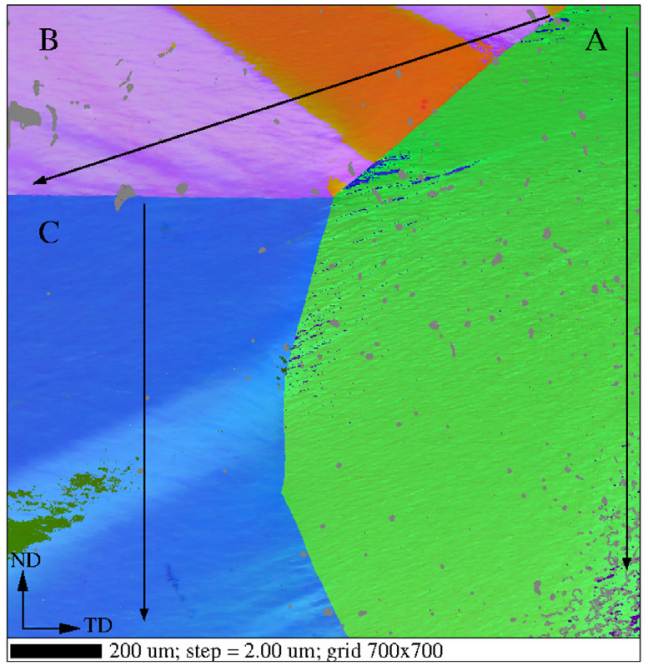

(a)
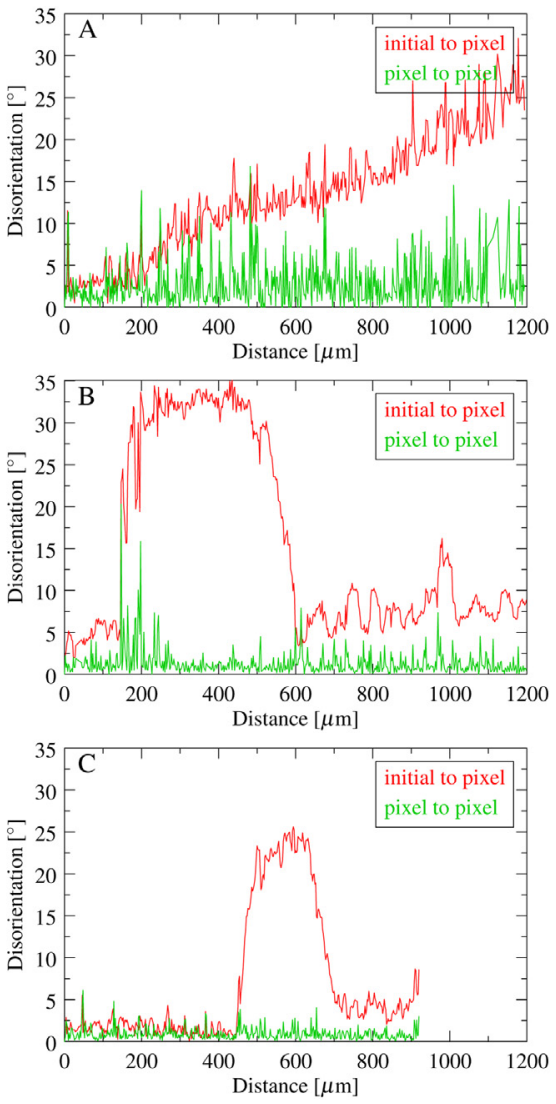

(b)
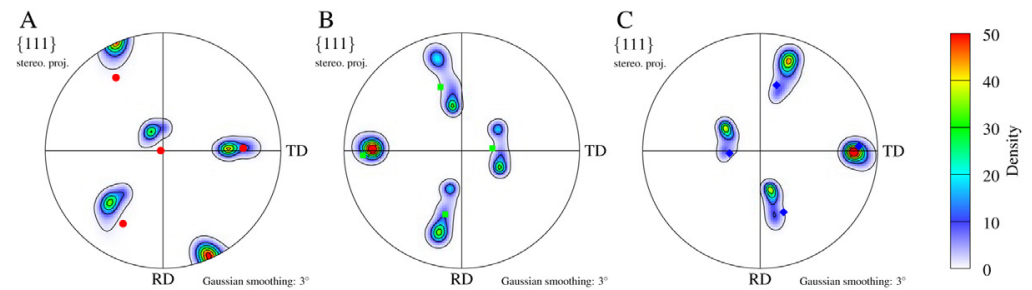

(c)

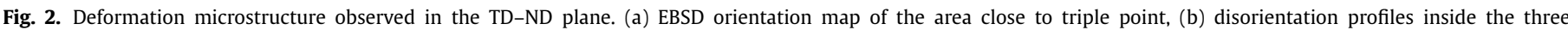

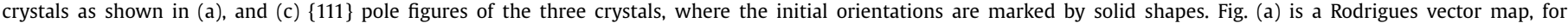
which Rodrigues vectors, $\boldsymbol{r}$, of components $\boldsymbol{r}_{1,2,3}$, are mapped to the RGB colour space as $\{R, G, B\}=\left\lfloor 255\left(r_{i}+\sqrt{2}-1\right) /[2(\sqrt{2}-1)]\right\rfloor$.

of the slice). The top region of the map belongs to crystal B while the rest of the map belongs to crystal A. Focus is on crystal A, which appears significantly subdivided and shows different local orientation fields along ND. This can first be seen from the disorientation profile plotted in Fig. 3b. The top part exhibits particularly large local disorientations, up to $45^{\circ}$ over a distance of $1 \mathrm{~mm}$, while the rest of the crystal has smaller disorientations, of the order $10-15^{\circ}$. The different magnitudes of disorientations can likely be attributed to the different deformations resulting from grain interaction: the top part of crystal A is closer to the grain boundary and therefore likely to be more affected by grain interaction, and Fig. $2 \mathrm{a}$ also shows that this region is close to the transition band of crystal B. This interpretation was confirmed by a complementary experiment, for which a single crystal of the same orientation as crystal A was deformed under the same conditions as the tricrystal and showed only limited local disorientations, as does the bottom part of crystal A.

To further investigate the change in orientation field in crystal A along ND, three regions of size $225 \mu \mathrm{m} \times 225 \mu \mathrm{m}$ were considered, AI, AII and AIII, as shown in Fig. 3a. The orientation distri- butions in these three regions, which are shown in Fig. 3c, differ significantly in terms of how much they extend preferentially in specific directions, i.e. their "anisotropy". The anisotropy of an orientation set can be described using metrics first proposed by Glez and Driver [15] and Barton and Dawson [16]. The average orientation being computed using quaternions [15], the disorientation of each orientation, with respect to the average orientation is written as the disorientation vector, $\boldsymbol{w}=\boldsymbol{r} \theta / 2$, where $\boldsymbol{r}$ and $\theta$ are the disorientation axis and angle, respectively. In this work, the disorientation vector was used instead of the Rodrigues vector [13-15] or the imaginary part of a quaternion [17], as it results from exponential mapping of disorientations in the tangent space of orientation space, which is a space particularly appropriate for analysing disorientation distributions $[18,19]$. This choice is, however, only rooted in theoretical considerations and does not affect the results significantly. Given a disorientation set, $\boldsymbol{w}^{\alpha}(\alpha=1, \ldots, N)$, a $3 \times 3$ covariant matrix, $\boldsymbol{S}$, is defined as

$\boldsymbol{S}=\frac{1}{N} \sum_{\alpha=1}^{N}\left(\boldsymbol{w}^{\alpha} \otimes \boldsymbol{w}^{\alpha}\right)$, 


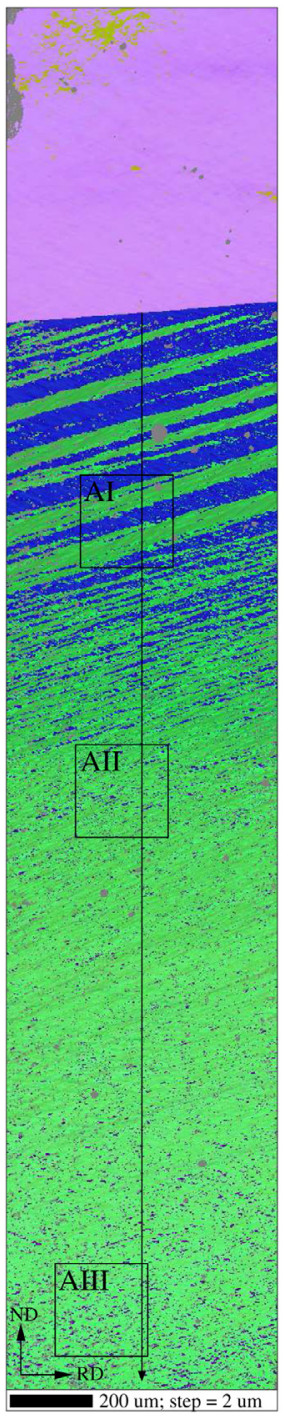

(a)

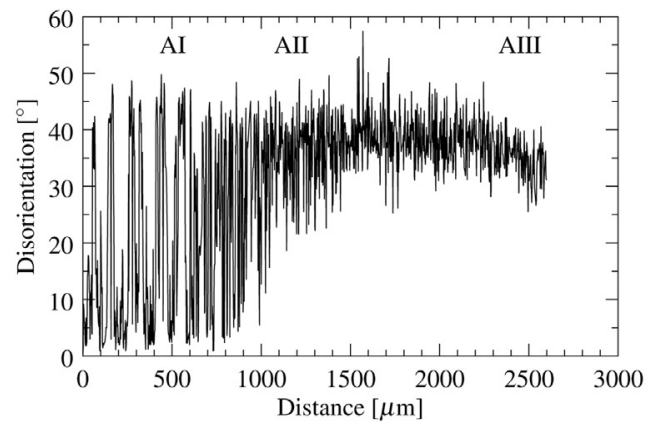

(b)
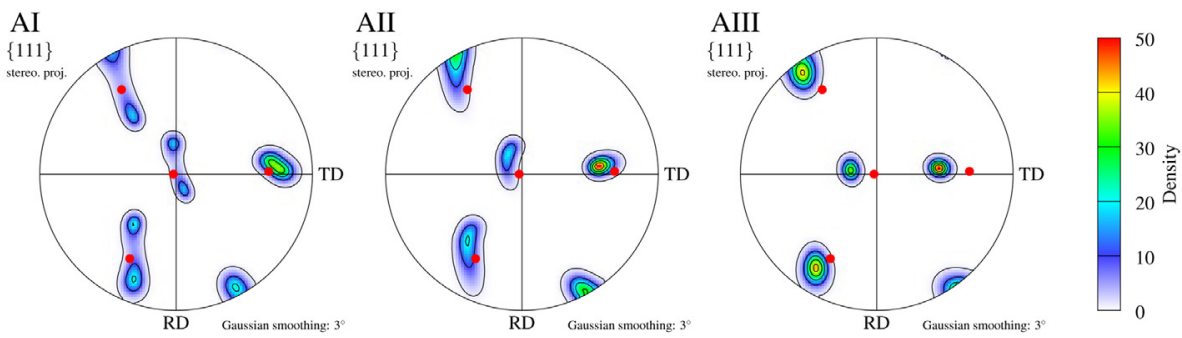

(c)

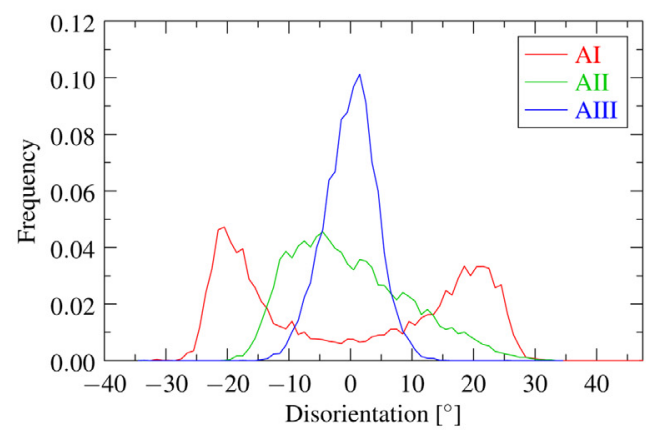

(d)

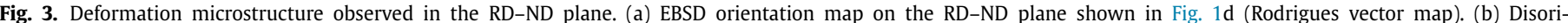

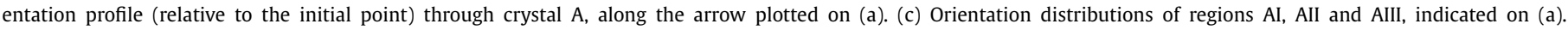
(d) Disorientation distributions about the preferential disorientation axis $\left(\theta_{i}\right.$, about $\left.v_{i}\right)$, for regions AI, AII and AIII.

which is symmetric and can be diagonalized. The eigenvectors, $\boldsymbol{v}_{\boldsymbol{i}}$ $(i \in\{1,2,3\})$, and the square roots of the eigenvalues, $\theta_{i}\left(\theta_{1} \geq \theta_{2} \geq\right.$ $\left.\theta_{3}\right)$, provide the principal axes and characteristic lengths of the distribution, respectively. The first principal axis $\left(\boldsymbol{v}_{\mathbf{1}}\right.$, associated to $\left.\theta_{1}\right)$ corresponds to the disorientation axis about which the disorientation angles are the highest and will be referred to as "preferential disorientation axis" in the following [14]. The angular components of the disorientation vectors along the principal axes, $\theta_{i}^{\alpha}$, can be obtained as

$\theta_{i}^{\alpha}=2 v_{i} \cdot \boldsymbol{w}^{\alpha}$.

The characteristic lengths of the distributions $\left(\theta_{i}\right)$ are also equal to their standard deviations. Crystals deformed in plane strain compression (or rolling) typically show a preferential disorientation axis close to TD $[14,15,17]$. As discussed previously for crystals A, B and $C$ and shown in the following for regions AI, AII and AIII, the present experimental findings make no exception to these observations. Regions AI, AII and AIII have a preferential disorientation axis $\left(v_{1}\right)$ within $16^{\circ}, 23^{\circ}$ and $27^{\circ}$ of $\mathrm{TD}$, respectively. However, regions AI, AII and AIII have appreciably different standard deviations of the disorientation angles about the preferential disorientation axis $\left(\theta_{1}\right.$, about $\left.\boldsymbol{v}_{\mathbf{1}}\right)$, of $17.8^{\circ}, 9.6^{\circ}$ and $4.3^{\circ}$, respectively, while the standard deviations of the disorientation angles about the two other axes $\left(\theta_{2,3}\right.$, about $\left.\boldsymbol{v}_{\mathbf{2}, 3}\right)$ have similar values of $2-3^{\circ}$. So, there is a clear trend toward smaller disorientation angles about the preferential disorientation axis $\left(\theta_{1}\right.$, about $\left.\boldsymbol{v}_{\mathbf{1}}\right)$ when moving across crystal A from AI to AIII, i.e. along -ND. The $\theta_{1}^{\alpha}$ distributions are plotted, for the three regions, in Fig. 3d. While regions AII and AIII show typical unimodal, approximately-Gaussian distributions, region AI shows a bimodal distribution, which corresponds to orientation fragmentation [13-15].

\subsection{Substructures}

The substructures of regions AI, AII and AIII are shown in Fig. 4 as disorientation maps, on which colour represents the disorientation vector. The disorientations are computed with respect to the average orientation of the map (except for region AI, for which the reference orientation is located halfway between the two orientation modes (as visible in Fig. 3c), for better rendering). Using such 

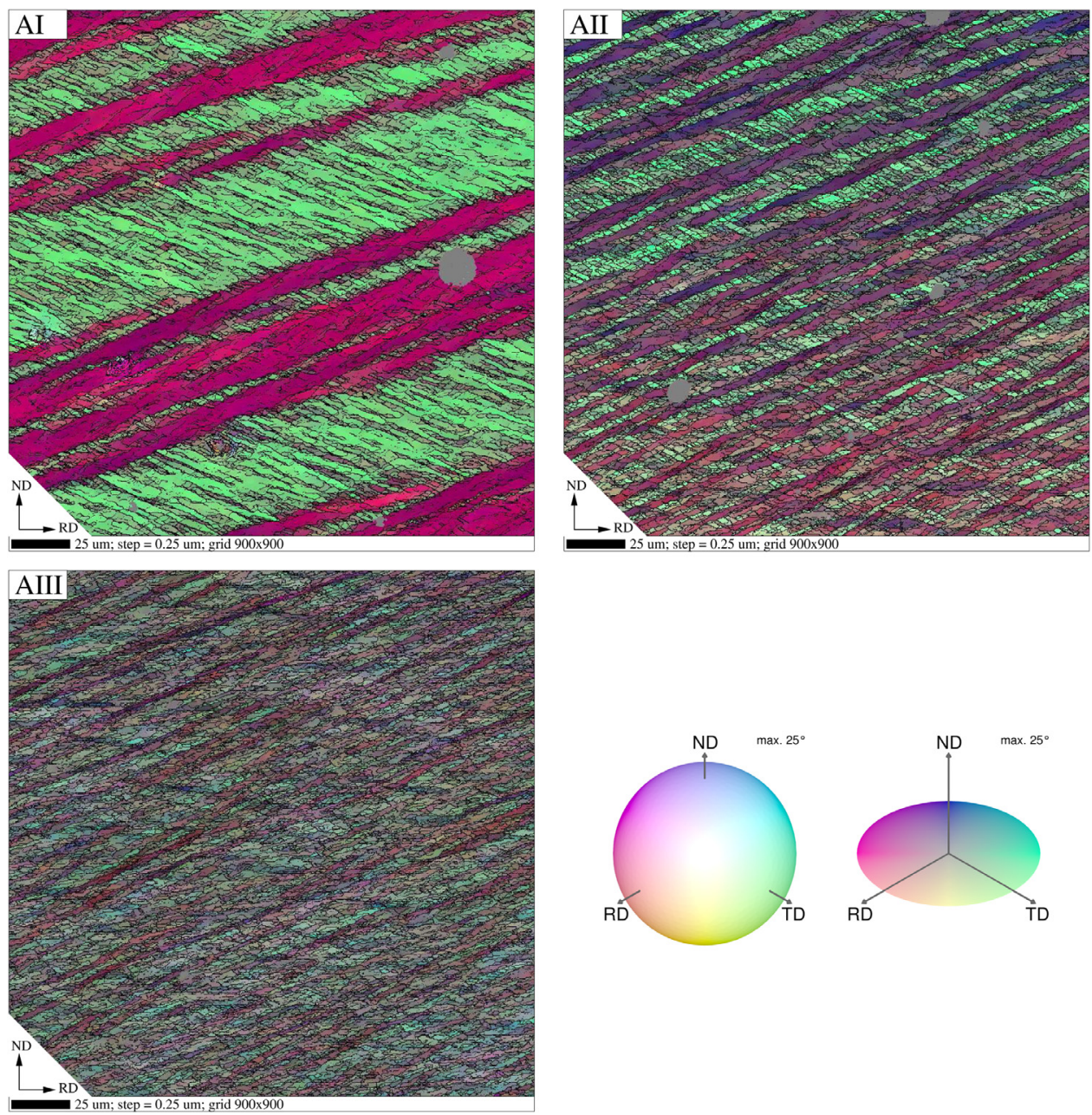

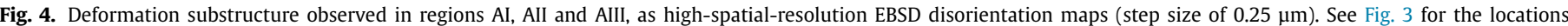

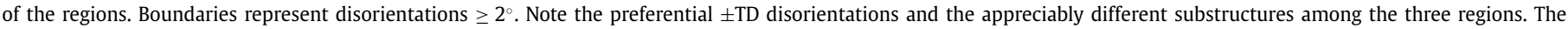

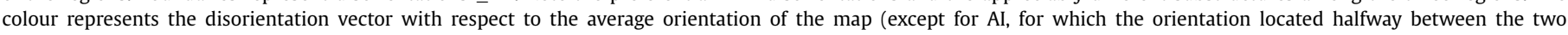
orientation modes of Fig. $3 \mathrm{c}$ is used).

a parameterization, all disorientations within a given angle, $\theta_{\max }$ (here, $25^{\circ}$ ), are contained within a ball of radius $\theta_{\max } / 2$, and their components feed the RGB colour channels to produce the colour key of Fig. 4. The main advantages of such a colour key are that the preferential disorientations can be readily identified, and that disorientations about opposite axes (or the same axis but opposite directions) appear in complementary RGB colours, which straightforwardly reveals the band structures with alterning orientations that typically develop in deformed crystals. In Fig. 4, all substructures exhibit shades of green and magenta, which correspond to disorientations preferentially distributed about $+\mathrm{TD}$ and $-\mathrm{TD}$, respectively (or, equivalently, about +TD but in opposite directions), as was reported in Section 3.1. However, it is clear that the different orientation distributions of regions AI, AII and AIII (see Fig. 3b) correspond to different substructures (see Fig. 4). First, it appears that high disorientation angles about the preferential disorientation axis $\left(\theta_{1}^{\alpha}\right.$, about $\left.\boldsymbol{v}_{\mathbf{1}}\right)$ or, equivalently, a high $\theta_{1}$ value, such as those of AI and AII, lead to a "sharp" substructure, composed of distinct bands with large disorientations with respect to each other. In contrast, the low disorientation angles $\left(\theta_{1}^{\alpha}\right)$ of region AIII lead to a diffuse substructure. Second, the substructures can be analysed in more detail. The "fragmented" orientation distribution of region AI corresponds to a multiscale substructure composed of $50-\mu \mathrm{m}$-thick deformation bands of nearly homogeneous orientation, rotated from the average orientation by about $20^{\circ}$ but in opposite directions (+TD and -TD) from one band to the next, subdivided into finer bands, corresponding to smaller rotations about $\pm \mathrm{TD}$. Throughout the structure, the $+\mathrm{TD}$ rotated bands appear nearly homogeneous while the -TD-rotated bands contain small inserts of +TD-rotated regions (in a different plane). Region AII does not exhibit such a multiscale substructure but rather is composed of +TD-rotated and -TD-rotated bands, although some substructuring can still be seen in the +TD-rotated bands. Region AIII has a subtructure qualitatively similar to region AII, but it is much more diffuse.

\subsection{Stored energy}

The stored energy associated with the dislocation boundaries can be estimated from the high-spatial-resolution EBSD orientation maps of regions AI, AII and AIII provided in Fig. 4, using a method similar to that proposed by Godfrey et al. [10]. For medium and high stacking fault energy materials, such as the aluminium used in this work, the dislocation density inside the dislocation cells is small compared to the dislocation density of the dislocation boundaries and can be neglected to first order [10]. The stored energy per unit volume, $E$, can be calculated by summing the contributions of all dislocation boundaries. In an EBSD map, the dislocation boundaries are taken as the pixel boundaries of disorientation angles higher than a specific threshold, $\theta_{\min }$. The stored energy, $E$, 


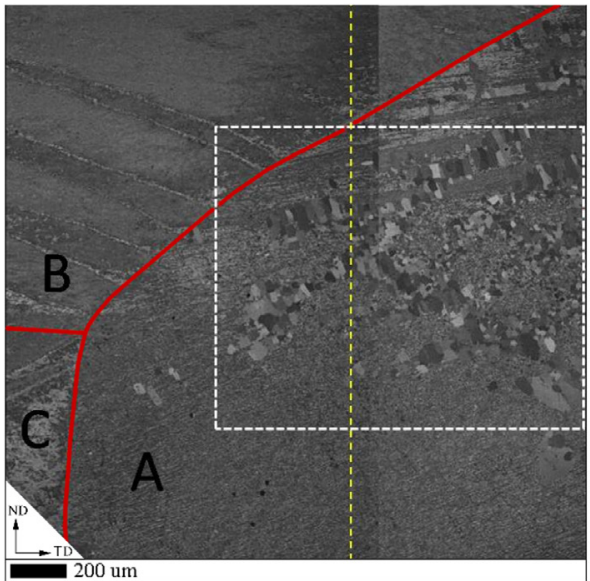

(a)

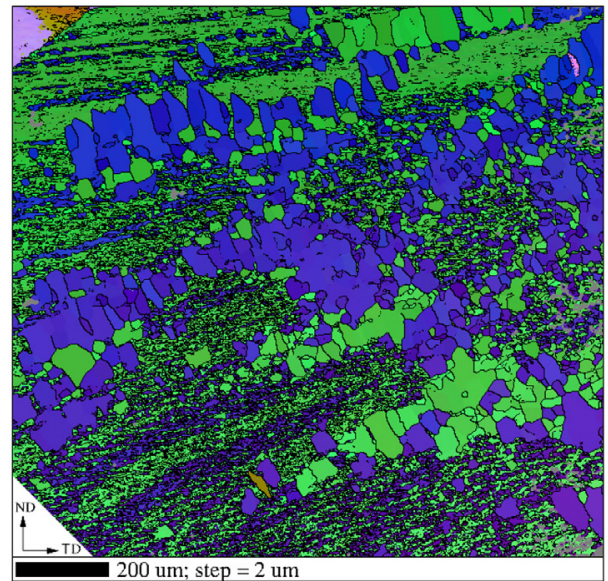

(b)

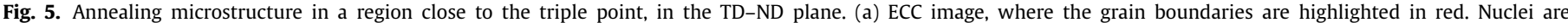

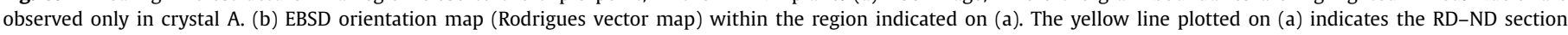
used in Fig. 6.

can therefore be written as

$E=\frac{C \Delta}{A} \sum_{\alpha} \gamma\left(\theta^{\alpha}\right)$

where $\theta^{\alpha}$ is the disorientation angle of a pixel boundary, $\Delta$ and $A$ are the step size and surface area of the EBSD orientation map, respectively, $C=\pi / 4$ accounts for the fact that the boundary length per unit area may be overestimated due to the stepped shape of the boundaries in the EBSD orientation map, and $\gamma\left(\theta^{\alpha}\right)$ is the stored energy per unit area given by the Read-Shockley equation [20],

$\gamma\left(\theta^{\alpha}\right)=\gamma_{m} \frac{\theta^{\alpha}}{\theta_{m}}\left(1-\ln \frac{\theta^{\alpha}}{\theta_{m}}\right)$.

We use $\theta_{m}=15^{\circ}, \gamma_{m}=0.324 \mathrm{~J} . \mathrm{m}^{-2}$ [21] and $\theta_{\min }=1.5^{\circ}$. The resulting values of the average stored energy are $0.22,0.28$ and $0.27 \mathrm{MJ} . \mathrm{m}^{-3}$ for regions AI, AII and AIII, respectively. The stored energy therefore varies only moderately between regions AI, AII and AIII, despite their appreciably different substructures (see Section 3.2). It is remarkable that the stored energy is the smallest in region $\mathrm{AI}$, which is a highly-fragmented region.

\section{Annealing microstructure}

The annealing microstructure is analysed in slice 3 (see Fig. 1). As in the case of the deformation microstructure, focus is on the region near the triple line as well as along the full height of crystal A. We analyse first the orientations of the nuclei and second their density, and we compare them to the stored energies of regions AI, AII and AIII.

\subsection{Matrix-to-nuclei orientation relationship}

The microstructure in an area close to the triple point, in the TD-ND plane, is shown in Fig. 5. It can be seen in Fig. 5a that, under the investigated annealing conditions, recrystallisation occurred in crystal A but not in crystals B and C, despite the presence of deformation bands in these two crystals (this was actually confirmed by observations on the whole TD-ND plane). Moreover, no nuclei were observed at the grain boundaries nor at the triple point. Although such sites often are observed to stimulate nucleation in aluminium, detailed studies of the deformation microstructures at regions near original grain boundaries and triple junctions have revealed that, depending on the crystallographic orientations of the grains, strong orientation gradients or high local stored energies may or may not develop near these sites $[22,23]$. For the present sample, the deformation microstructures near the boundaries are similar to those in the bulk of the grains, and therefore grain boundary nucleation is not dominating.

The orientations of the nuclei are clearly related to those of the deformation microstructure (or "matrix"), as seen from the EBSD orientation map provided in Fig. 5b, and the nuclei are somewhat preferentially elongated in a direction normal to the deformation bands, which indicates that they grow toward the surrounding bands.

To analyse the relationship between the orientations of the nuclei and those of the matrix, the microstructure was mapped in the RD-ND plane (along the yellow line of Fig. 5a), as illustrated in Fig. 6. The map corresponds to the same area as the one of the deformation microstructure of Fig. 3, which enables direct comparison (although the two maps where acquired on different sample slices). Again, nuclei are only found in the narrow deformation bands and transition bands located in region AI (extending toward region AII), but not at the triple line, grain boundaries nor wide deformation bands. The relationship between the orientations of the nuclei and those of their parent sites is investigated using three typical regions of the map of crystal A shown in Fig. 6a, which contain both nuclei and remainders of the deformation matrix, are located between regions $\mathrm{AI}$ and $\mathrm{AII}$, and are referred to as $\mathrm{AI}^{\prime}, \mathrm{AI}^{\prime \prime}$ and $\mathrm{AI}^{\prime \prime \prime}$. The three regions have a size of $600 \mu \mathrm{m} \times 280 \mu \mathrm{m}$, which is both sufficiently small to contain a fairly uniform deformation microstructure and sufficiently large to provide representative results. In particular, the orientation distribution of the remainder of the deformation matrix inside a region can be considered as qualitatively representative of that of the full (parent) deformation matrix in that region, which is confirmed to be reasonable both by their similarity with the orientation distribution of the deformation microstructure of region $\mathrm{AI}$ shown in Fig. $3 \mathrm{c}$ and their evolution toward less intense orientation fragmentation when moving along $-\mathrm{ND}$, as also observed in Fig. 3c. The individual nuclei were detected automatically using three criteria: a diameter of the circle of equivalent surface area larger than $8 \mu \mathrm{m}$, an internal average disorientation angle with respect to the average orientation smaller than $1.5^{\circ}$ and the fact that they are surrounded by at least one high angle boundary $\left(\geq 15^{\circ}\right)$. The orientation distributions of the matrix and nuclei are provided as $\left\{\begin{array}{lll}1 & 1 & 1\end{array}\right\}$ pole figures in Fig. $6 \mathrm{~b}$. All pole 

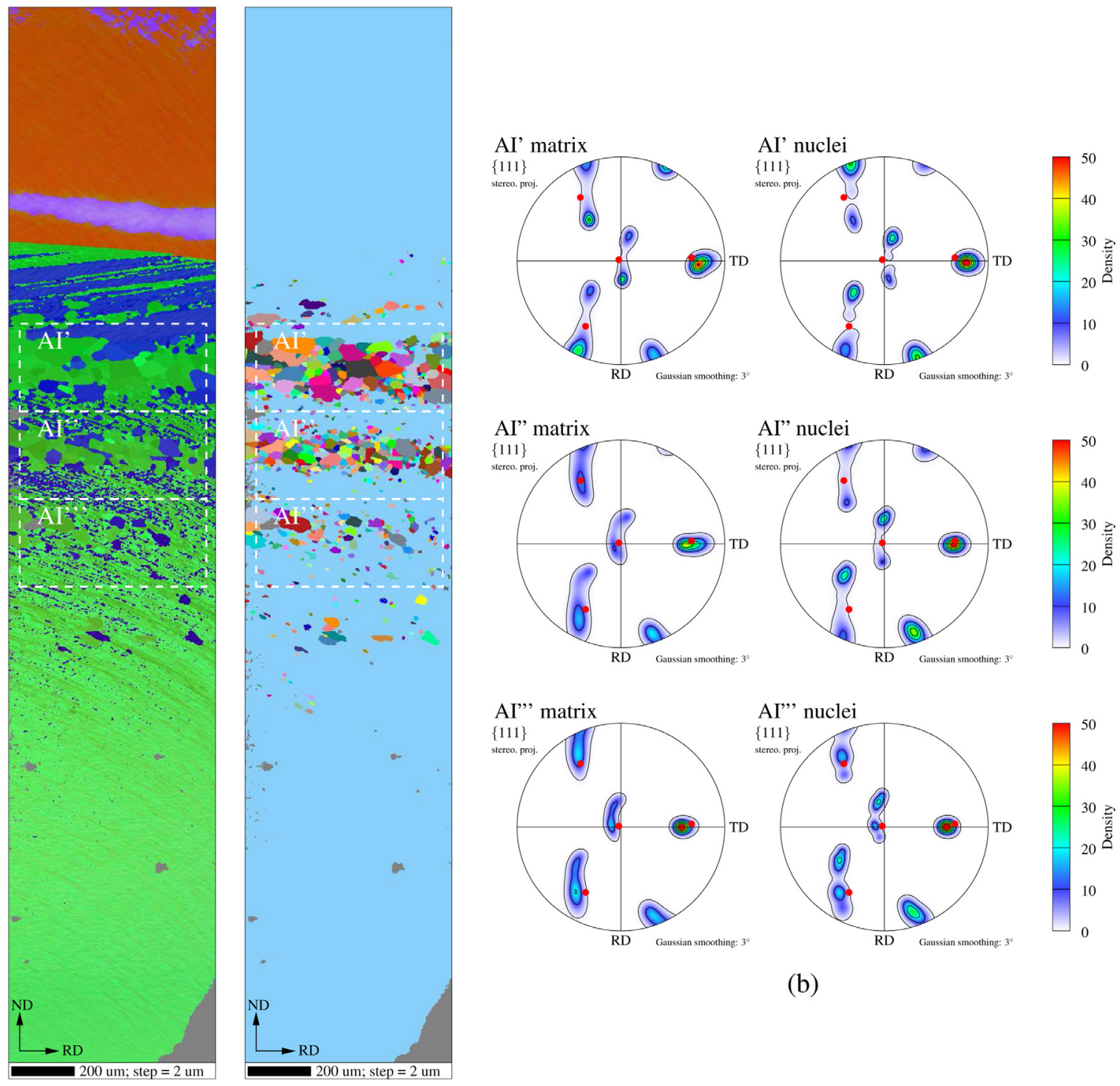

(b)

(a)

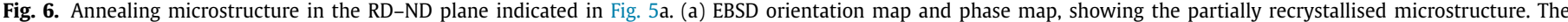

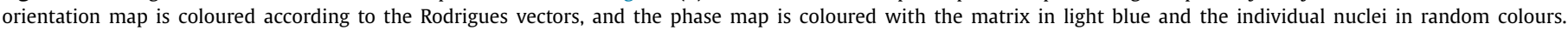
(b) $\{111\}$ pole figures of the deformation matrix and of the nuclei inside regions $\mathrm{AI}^{\prime}, \mathrm{AI}^{\prime \prime}$ and $\mathrm{AI}^{\prime \prime \prime}$ of (a), respectively.

figures are plotted in terms of pole density, which is computed by assigning a Gaussian kernel (half-width of $3^{\circ}$ ) to the $\left\{\begin{array}{lll}1 & 1 & 1\end{array}\right\}$ poles of each orientation. First, the pole figures clearly show that the orientations of the nuclei are among the orientations of their parent deformation matrix, which corresponds to nucleation arising by subgrain growth, a phenomenon previously observed in 3D [6]. Second, the pole figures (and so the orientation distributions) can also be compared quantitatively. As pointed out above, each orientation is actually represented by a (small) Gaussian distribution, which somewhat smoothes the distributions, but since the same kernel is used for both the orientation distributions of the matrix and the nuclei, it is possible to compare their intensities (so as the location of their maxima) quantitatively, without actually introducing bias. (At the opposite, considering a (smoothed) orientation distribution for the matrix but individual orientations for the nuclei would not enable a quantitative comparison.) As seen in Fig. 6b, the nuclei systematically show more "bimodal" orientations distributions than the matrix, with peaks that are more intense and located further from the average. This indicates that the orientations of the nuclei are preferentially located at the "tails" or "outskirt" of the orientation distributions of the deformation matrix. Among all the orientations of the distribution, these orientations are those that show the highest disorientations with respect to the rest of the orientations. They are therefore also likely to be the orientations surrounded by the highest local disorientations in the microstructure.

\subsection{Nucleation sites}

Nucleation occurred in crystal A, which is the crystal of maximum Taylor factor but also, and more interestingly, the crystal of maximal stored energy. This can first be established from the TDND orientation map of the deformation microstructure shown in Fig. 2. The average stored energy of each of the crystals can be computed in the same way as in Section 3.3, where it was done from the (RD-ND) high-spatial-resolution maps. When done from coarser maps, artefacts are to be expected since some dislocation boundaries are missed [24,25]. It is still possible, however, to use 
the orientation map shown in Fig. 2 for a semi-quantitative evaluation. The average stored energies obtained for crystal $A, B$ and $C$ are equal to $0.084,0.034$ and $0.013 \mathrm{MJ} \cdot \mathrm{m}^{-3}$, respectively. Crystal A therefore has the maximum stored energy. It is also possible to establish that crystal A has the highest average stored energy from the crystal plasticity theory. First, let us recall that the Taylor factor relates the microscopic properties and the macroscopic properties through the power of plastic deformation, and that (assuming isotropic hardening) it can be written as $M=\sum_{\alpha} \dot{\gamma}^{\alpha} / \overline{\dot{\varepsilon}}$, where $\dot{\gamma}^{\alpha}$ are the slip rates and $\overline{\dot{\varepsilon}}$ is the equivalent plastic strain rate. Then, let us note that the tricrystal morphology and the orientations of the three crystals are such that the three crystals actually undergo about the same (normal) deformation, which corresponds to the applied deformation. Indeed, crystal A occupies the whole sample thickness and is therefore subjected to the imposed deformation, and crystals B and C, although they are "in series" along ND, have equivalent orientations with respect to ND, and so deform equally under compression along ND, i.e. according to the imposed deformation. It can be concluded that crystal A has the highest slip rates $\left(\sum_{\alpha} \dot{\gamma}^{\alpha}\right)$ by a factor equal to the ratio between the Taylor factors, i.e. $3.7 / 2.4 \simeq 1.5$, and so also the highest resolved shear strengths, dislocation densities, and finally (average) stored energy.

\section{Discussion}

The stored energy is generally considered and used as a criterion for recrystallisation nucleation. In this experiment, nucleation was found to occur in the crystal of maximum average stored energy; however, and more interesting, the local density of nuclei was found not to correlate with the local stored energy in the crystal, as most nuclei were found where the stored energy is the lowest. It is therefore of interest to devise a new (energy) criterion that would be able to describe our experimental observations.

It is clear from the results of Sections 3 and 4 that crystal A has very different substructures and orientation distributions along $\mathrm{ND}$, and that these differences are responsible for the different densities of nuclei. Our objective therefore is to integrate the properties of the local orientation distributions into the energy criterion. This can be done by comparing quantitatively the orientations map of the deformation microstructure shown in Fig. 3 and the orientation map of the annealing microstructure shown on Fig. 6, which represent the same area before and after annealing (although on different sample slices).

\subsection{Sliding-box analysis}

The general methodology to relate the local properties of the deformation microstructure of Fig. 3 to the local density of nuclei of the annealing microstructure of Fig. 6 is to evaluate properties, of each of the two maps, within a sliding box, which leads to property profiles similar to the disorientation profiles shown on Figs. $2 b$ and $3 \mathrm{~b}$, and to compare these profiles and their correlation. A box of size $660 \mu \mathrm{m} \times 220 \mu \mathrm{m}$ (i.e., the same height as AI, AII and AIII, and full width) is slid along the arrow plotted in Fig. 3a (considering the centre of the box as control point and excluding the first and last $110 \mu \mathrm{m}$ of the arrow).

First, from the orientation map of the annealing microstructure, the density of nuclei, $\rho_{n}$, is obtained by dividing the number of nuclei located inside the sliding box by its surface area, where nuclei that are only part of the box are counted based on the surface area fraction located inside the box. Results are provided on Fig. $7 \mathrm{a}$, and it can be seen that the density of nuclei $\left(\rho_{n}\right)$ shows a gradual decrease along -ND to reach a value of $0 \mathrm{~mm}^{-2}$ halfway along -ND. The density profile plotted in Fig. 7 shows an evolution smoother than expected visually from the map of Fig. 6, which exhibits several horizontal bands of nuclei. This is due to the fact that the sliding box has a height similar to the spacing between these bands and, in this work, is intentionally used so as to establish trends along ND rather than to capture fine details that cannot necessarily be interpreted (due to the uncertainties associated with the observation of similar, but yet different sample slices).

Second, from the orientation map of the deformation microstructure, the stored energy inside the sliding box $(E)$ can be computed. As already pointed out in Section 4.2, differences are to be expected with respect to the high-spatial-resolution maps shown on Fig. 4 due to a larger step size. Specifically, the stored energies of AI, AII and AIII are decreased from 0.22, 0.28 and $0.27 \mathrm{MJ} . \mathrm{m}^{-3}$ for the high-spatial-resolution map, respectively, to $0.12,0.15$ and $0.12 \mathrm{MJ} \cdot \mathrm{m}^{-3}$ for the standard-spatial-resolution map, respectively. The decrease is slightly higher for AIII, which has the finest and most diffuse microstructure and therefore more "missed" dislocation boundaries with the largest step size. However, it is still possible to use the orientation map of Fig. 3 for semi-quantitative stored energy evaluations, while the results on the orientation distributions that will be provided in Section 5.2 almost do not depend on the step size and remain fully quantitative. Results are provided in Fig. 7, from which it can be concluded that there is no correlation between the stored energy and the density

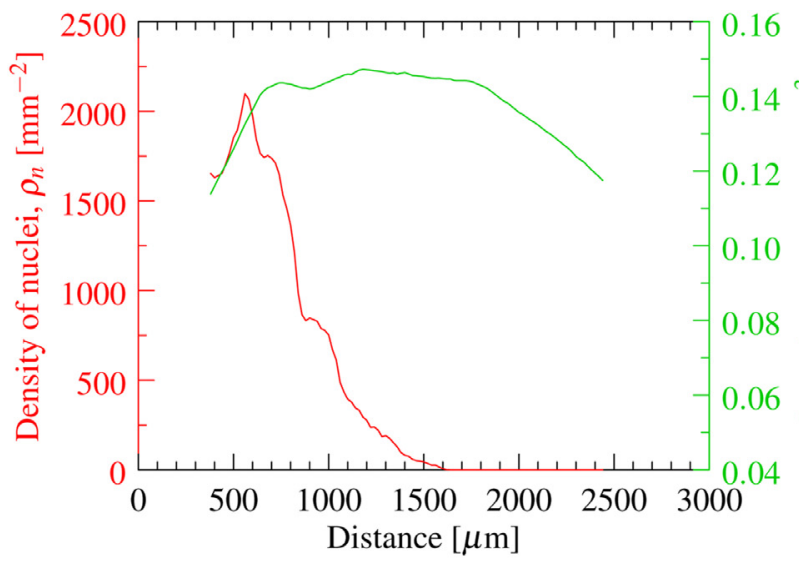

(a)

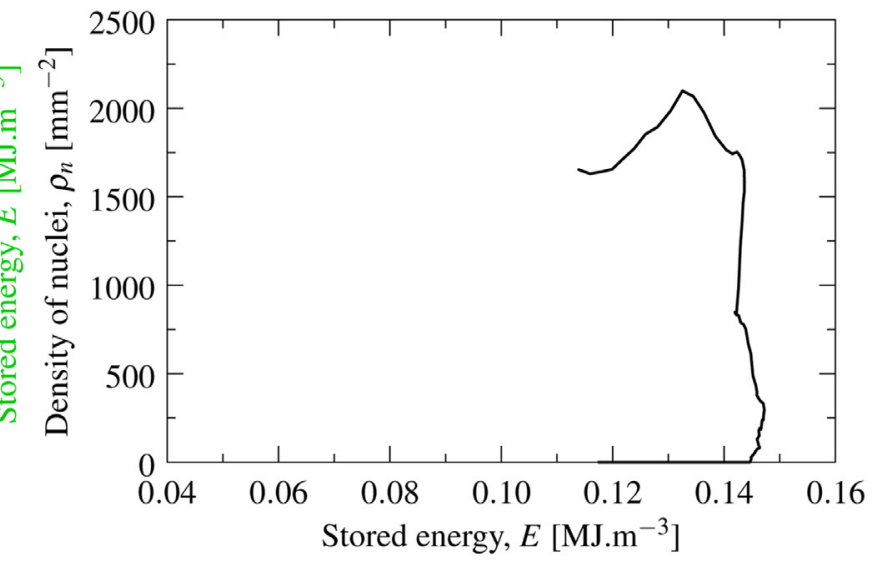

(b)

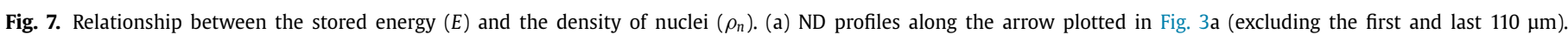
(b) Correlation between $E$ and $\rho_{n}$. 


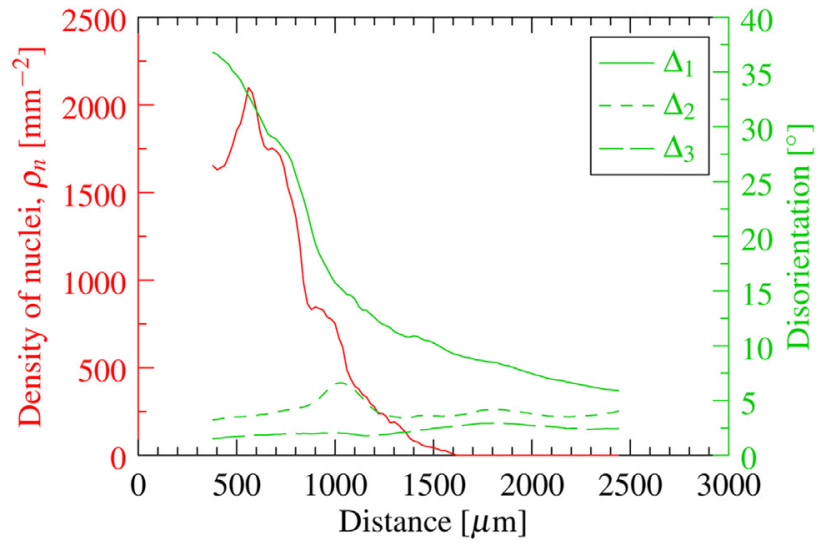

(a)

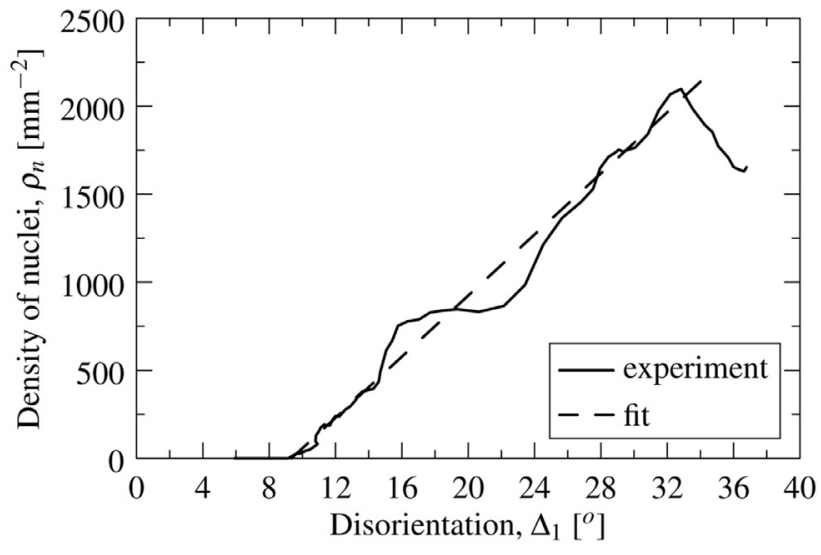

(b)

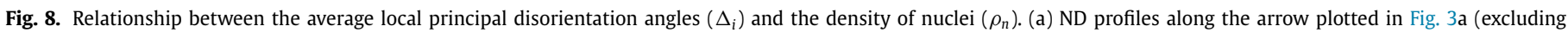
the first and last $110 \mu \mathrm{m})$. (b) Correlation between $\Delta_{1}$ and the density of nuclei $\left(\rho_{n}\right)$. Fit of the form $\rho_{n}=a\left(\Delta_{1}-b\right)$ with $a=86.8^{\circ} . \mathrm{mm}^{-2}$ and $b=9.35^{\circ}$.

of nuclei in crystal A, as similar stored energies can yield very different densities of nuclei, and different values of the stored energy can yield similar densities of nuclei. As a consequence, the stored energy cannot be considered, at least in this experiment, as a reliable criterion for recrystallisation nucleation.

\subsection{Determination of a new energy criterion}

The stored energy associated to the dislocation boundaries $(E)$, computed from the local disorientations, can be considered as a first-order criterion for recrystallisation nucleation. However, it does not directly include information such as the level of subdivision (into bands) of a crystal at the micrometer scale, or equivalently the anisotropy properties of the orientation distributions. In the following, we propose to devise a new energy criterion for recrystallisation nucleation that encompasses both the stored energy and the anisotropy of the orientation distribution as

$E_{p}=E f$,

where, as previously, $E$ is the stored energy of the microstructure, and $f \leq 1$ depends on the anisotropy properties of the orientation distribution. The values of both $E$ and $f$ are to be evaluated globally, over regions larger than the characteristic length of the substructure, but within which the substructure is still fairly uniform (such as regions $\mathrm{AI}, \mathrm{AII}$ and $\mathrm{AIII}$, or $\mathrm{AI}^{\prime}, \mathrm{AI}^{\prime \prime}$ and $\mathrm{AI}^{\prime \prime \prime}$ ). To determine the expression of $f$, we must first analyse the correlation between the anisotropy of the orientation distribution and the density of nuclei $\left(\rho_{n}\right)$.

\subsubsection{Influence of the local orientation distribution}

The evolution along ND of the orientation (or disorientation) distribution within the deformation microstructure and its relationship with that of the density of nuclei is analysed using the sliding box (as in Section 5.1), from the orientation map shown in Fig. 3a. The description of the anisotropy of a disorientation distribution by its $\theta_{i}$ (and $\boldsymbol{v}_{\boldsymbol{i}}$ ) values is only appropriate if the corresponding disorientation distributions about the principal disorientation axes $\left(\boldsymbol{v}_{\boldsymbol{i}}\right)$ are unimodal and approximately Gaussian, which is not everywhere the case inside crystal A (especially in its top part). In this work, we aim at describing the typical local disorientation angles of the substructure, as they locally drive the growth of nuclei, but these are not necessarily well described by the $\theta_{i}$ values. Instead, we define and use the average local principal disorientation angles, which can be estimated from the $\theta_{i}^{\alpha}$ distributions under the assumption that local disorientations form between orientations that alternate between the "left" and "right" parts of the distributions. This assumption is justified by the typical presence of alternating $\pm \mathrm{TD}$ orientations between adjacent bands, as shown in Fig. 4. The values of $\Delta_{i}$ can actually be computed as described in Appendix A. For a unimodal, Gaussian distribution, $\Delta_{i}=1.35 \theta_{i}$, while for a bimodal distribution, $\Delta_{i}=2 \theta_{i}$.

The results on the average local principal disorientation angles $\left(\Delta_{i}\right)$ and the density of nuclei $\left(\rho_{n}\right)$ are provided in Fig. 8. $\Delta_{1}$ show particularly high values at the small distances, i.e. where orientation fragmentation occurs, and decreases continuously along the profile. In contrast, $\Delta_{2,3}$ are nearly constant (as would be $\theta_{2,3}$, since $\theta_{2,3}^{\alpha}$ distributions always remain unimodal and approximately Gaussian $[13,14])$. The correlation between $\Delta_{1}$ and $\rho_{n}$ is even more clearly visualised in Fig. 8b, where $\rho_{n}=0$ for $\Delta_{1} \lesssim 9^{\circ}$ and $\rho_{n} \propto \Delta_{1}$ for $\Delta_{1} \gtrsim 9^{\circ}$.

\subsubsection{Primary stored energy}

We propose to define the energy criterion introduced in Equation 5 as

$E_{p}=E \frac{\Delta_{1}}{\Delta_{1}+\Delta_{2}+\Delta_{3}}$.

and we call it "primary stored energy", where "primary" relates to the scaling with respect to the average local principal disorientation angle about the first principal disorientation axis $\left(\Delta_{1}\right.$, about $\left.v_{1}\right) . E_{p}$ takes the maximal value of $E$ for an infinitely anisotropic orientation distribution (1-D orientation distribution) and a minimal value of $E / 3$ for an isotropic orientation distribution $\left(\Delta_{1}=\right.$ $\Delta_{2}=\Delta_{3}$ or, equivalently, $\theta_{1}=\theta_{2}=\theta_{3}$ ). The expression of $E_{p}$ is obviously very simple but, as will be seen in the following, provides good results for this experiment. A factor $f$ (see Equation 5) of the form $\Delta_{1} /\left(\Delta_{1}+\Delta_{2}+\Delta_{3}\right)$ can be interpreted as considering a substructure composed of disorientations of "pure" $v_{1}, v_{2}$ or $v_{3}$ axes (rather than mixed axes), for which only the boundaries of disorientations about $v_{1}$, which are those located between bands, contribute to recrystallisation nucleation. Regions AI, AII and AIII show values of $f$ of $0.88,0.66$ and 0.48 , respectively, and values of $E_{p}$ of $0.19,0.19$ and $0.13 \mathrm{MJ} . \mathrm{m}^{-3}$, respectively.

In Fig. 9a, it is shown that, in contrast to the stored energy $(E)$, which varies only moderately along ND (see Fig. 7), the primary stored energy $\left(E_{p}\right)$ shows a clear evolution composed of a slight increase followed by a gradual decrease, from $0.12 \mathrm{MJ} . \mathrm{m}^{-3}$ down to $0.06 \mathrm{MJ} \cdot \mathrm{m}^{-3}$. (The profile of $\rho_{n}$ shows a peak at a coordinate of $570 \mu \mathrm{m}$, while the profile of $E_{p}$ shows a peak at a coordi- 


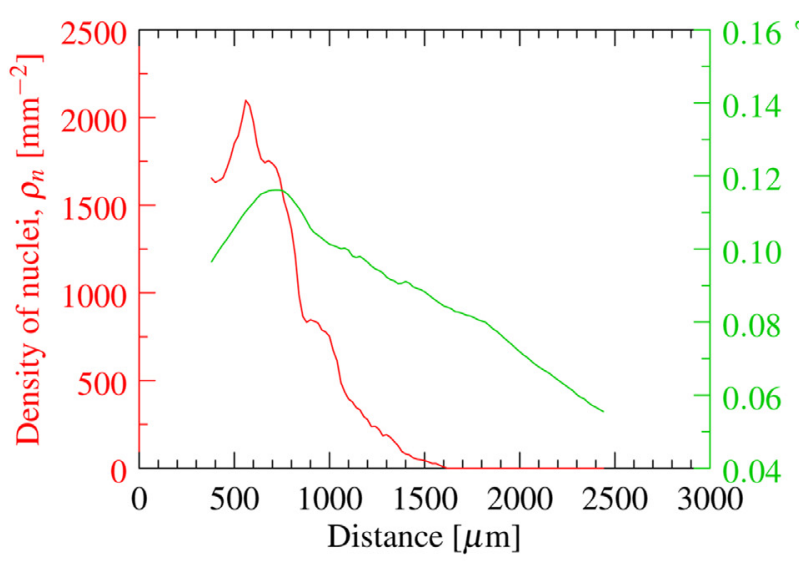

(a)

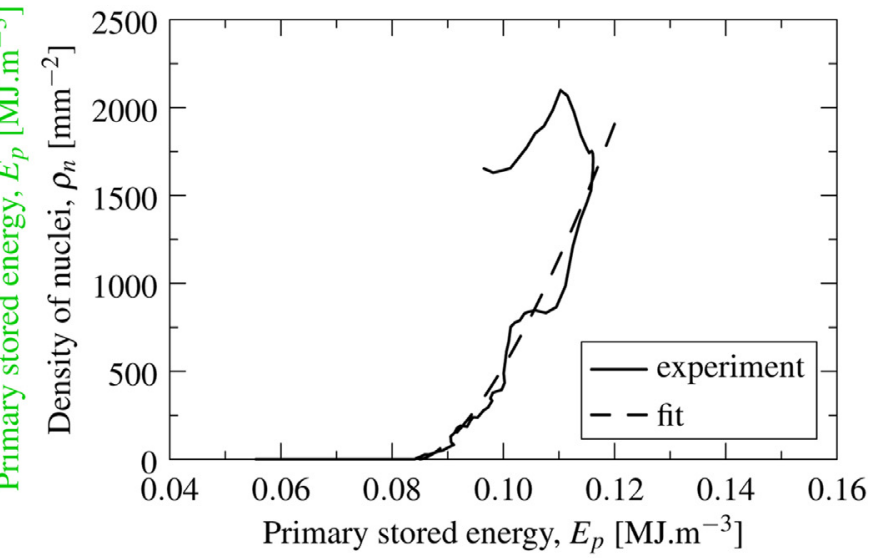

(b)

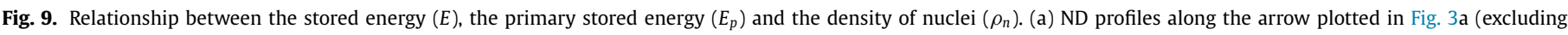

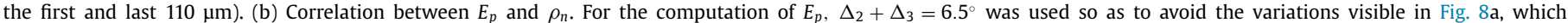

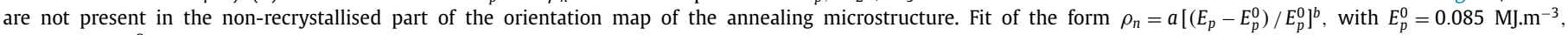
$a=7300 \mathrm{~mm}^{-2}$ and $b=1.5$.

nate of $720 \mu \mathrm{m}$. However, this mismatch can be reasonably attributed to the differences between the deformation microstructures of the respective sample slices. The hat shape apparent at high values on the curve of Fig. 9b, which results from the mismatch, is therefore simply discarded in the following.) The correlation between the primary stored energy $\left(E_{p}\right)$ and the density of nuclei $\left(\rho_{n}\right)$ is plotted in Fig. 9b and is quite clear: values of $E_{p}$ lower than $0.085 \mathrm{MJ} . \mathrm{m}^{-3}$ yield no nuclei, while values of $E_{p}$ above $0.085 \mathrm{MJ} . \mathrm{m}^{-3}$ yield increasing $\rho_{n}$ values. The density of nuclei $\left(\rho_{n}\right)$ at $E>0.085 \mathrm{MJ} . \mathrm{m}^{-3}$ can be described by a function of the form $\rho_{n}=a\left[\left(E_{p}-E_{p}^{0}\right) / E_{p}^{0}\right]^{b}$, with $E_{p}^{0}=0.085 \mathrm{MJ} \cdot \mathrm{m}^{-3}$ $a=7300 \mathrm{~mm}^{-2}$ and $b=1.5$. As mentionned in Section 4.2 , crystals $B$ and $C$ show comparatively lower values of the stored energy $(E)$ of 0.034 and $0.013 \mathrm{MJ} . \mathrm{m}^{-3}$, respectively (measured in the TD-ND section shown in Fig. 2). When measured at the top of the orientation map shown on Fig. 3, the energies of crystal B have even lower values of $E=0.008 \mathrm{MJ} . \mathrm{m}^{-3}$ and $E_{p}=0.0038 \mathrm{MJ} . \mathrm{m}^{-3}$. Crystals $B$ and $C$ therefore have values of the primary stored energy $\left(E_{p}\right)$ lower than $E_{p}^{0}$, which explains why they do not recrystallise in this experiment.

\subsection{Importance of the anisotropy of the orientation distribution for nucleation}

The classical approach to calculate the stored energy from orientation maps involves both the disorientation angles and the spacings between the dislocation boundaries [10], but not the anisotropy of the orientation distribution. However, orientation distributions with different anisotropies have different (frequency) distributions of the disorientation angles. Previous works showed that deformation microstructures with similar stored energies but different dislocation structures (in terms of the geometrical arrangements of the dislocation boundaries) have different nucleation behaviours [26,27]. As a matter of fact, a recent work based on 3D in situ observations [6] showed that the "embryos" of nuclei, which are already present in the deformation microstructure, are at least partly surrounded by high angle boundaries, which have higher energies but also higher mobilities than low angle boundaries. Our experimental observations come in general agreement with these previous works. The above analysis showed that the anisotropy of the orientation distribution, which is related to the disorientation angles of the dislocation boundaries but is accessible at a coarser scale, is an essential factor for nucleation, in addi- tion to the stored energy. By introducing the concept of "primary stored energy", it was possible to combine the effects of these several factors.

Orientation distributions inside individual deformed grains are anisotropic independently of the (average) orientation [14], but orientation fragmentation [13], which develops only for a small fraction of grains in conventional deformation modes [28], results in very large orientation anisotropies and therefore seems to offer particularly favorable recrystallisation nucleation conditions. Previous works have successfully integrated the anisotropy properties of the orientation distributions and orientation fragmentation into modelling, based on the orientation distributions over the entire grains [29]. This work suggests that the anisotropy of the orientation distributions could be considered at an even smaller scale.

\section{Conclusions}

The details of recrystallisation nucleation in a cold rolled aluminium tricrystal were related to the properties of the parent, deformation microstructure. Thanks to a similar deformation microstructure through the sample length, it was possible to slice it into several parts of similar deformation microstructures and carry out detailed investigations by going "back and forth" between the deformation and annealing microstructures. This enabled an indepth analysis that would not have been possible in a more conventional experiment. The main results are as follows:

- Recrystallisation preferentially developed in the crystal of highest average stored energy, but not at a priori presumed preferential locations such as triple lines or grain boundaries, nor at the location of highest local stored energy in that crystal.

- The orientations of the nuclei were among those of the parent, deformation microstructure, and were preferentially located at the outskirt of the parent orientation distribution. These orientations are likely to have the highest local disorientations in the deformation microstructure.

- The different densities of nuclei developed in regions of substructures composed of more or less sharp bands, and the band sharpness was found to be related to the anisotropy properties of the associated orientation distribution. This is important since the anisotropy properties of the orientation distributions can be measured at a scale coarser than that of the substructure. 
- The average local principal disorientations $\left(\Delta_{i}\right)$ were estimated from the disorientation distributions about the principal disorientation axes $\left(\theta_{i}^{\alpha}\right.$, about $\left.\boldsymbol{v}_{\boldsymbol{i}}\right)$, by considering that the orientations locally alternate between the "left" and "right" parts of the $\theta_{i}^{\alpha}$ distributions. For $\Delta_{1}$, this is in accordance with the fact that the adjacent bands have orientations rotated about the same axis $\left(\boldsymbol{v}_{\mathbf{1}}\right)$ but by opposite angles with respect to each other. This new metric $\left(\Delta_{i}\right)$ can be computed for any type of distribution (unimodal or not) and is not linearly related to the more usual standard deviation $\left(\theta_{i}\right)$.

- Regions with similar stored energies but different orientation distribution anisotropies, and especially $\Delta_{1}$ values, resulted in significantly different densities of nuclei after annealing. A linear relationship between $\Delta_{1}$ and the density of nuclei $\left(\rho_{n}\right)$ was found for $\Delta_{1}>10^{\circ}$. This documents that the orientation distribution anisotropy has to be considered for sound predictions of recrystallisation nucleation.

- A revised energetic criterion, named "primary stored energy" $\left(E_{p}\right)$, was proposed, which takes into account both the stored energy $(E)$ and the anisotropy properties of the orientation distribution $\left(\Delta_{i}\right)$. The density of nuclei $\left(\rho_{n}\right)$ showed a clear correlation with the primary stored energy $\left(E_{p}\right)$. Even if it was devised from observations from one columnar tricrystal only, we suggest that a nucleation criterion based on the primary stored energy (or criteria including the anisotropy properties of the orientation distribution in other ways) is the way forward when the aim is to predict active nucleation sites.

\section{Declaration of Competing Interest}

The authors declare that they have no known competing financial interests or personal relationships that could have appeared to influence the work reported in this paper.

\section{Acknowledgments}

The authors wish to dedicate this work to Julian Driver, who intiated this work several years ago and deceased while it was in progress. Julian has been collaborating for many years with members of the Risø/DTU group and has been a guest scientist for several months there. Julian has always been promoting exchanges between his group in Saint-Étienne and the Risø/DTU group. He has been an inspiration to many in both groups and has always been keen to help and involve young collaborators. Thank you Julian.

This work was partially funded by the European Research Council (ERC) under the European Union's Horizon 2020 research and innovation programme (M4D grant agreement No. 788567 ERC2018-ADG).

\section{Appendix A. Computation of the average local disorientation, $\Delta_{i}$}

Given a disorientation distribution along a principal disorientation axis $\left(\boldsymbol{v}_{\boldsymbol{i}}\right)$, the average local disorientation angle, $\Delta_{i}$, is computed by splitting the distribution in half, relative to the average, as exemplified in Fig. 10 for the $\theta_{1}^{\alpha}$ distributions of regions AI, AII and AIII. The underlying assumption is that the orientations of adjacent bands alternate between these two parts (i.e., switch from positive to negative values, and vice versa, of the distribution), which is typically encountered when the substructure is made of bands (Fig. 4). For a unimodal distribution, this procedure just splits the distribution, while for a bimodal distribution, it separates its two modes. The average values of the two parts (or modes) can then be computed, and the average local disorientation angle, $\Delta_{i}$, is taken as the distance (or disorientation) difference between the two modes. Of course, this assumes equal volume fractions of the two modes (which corresponds to alterning bands of the
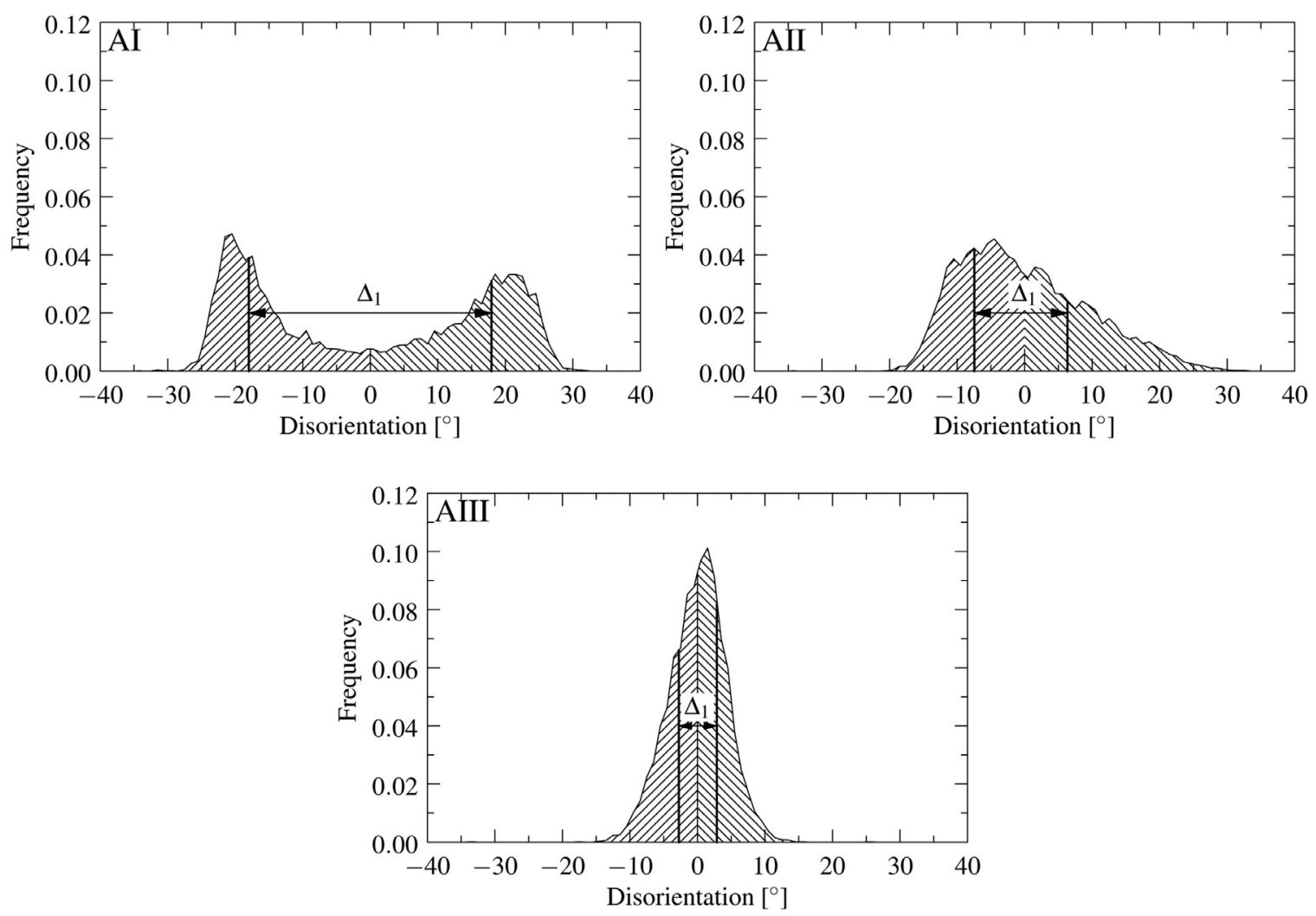

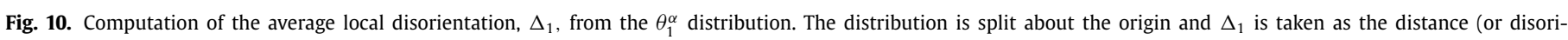
entation) between the two parts of the distribution. Examples are provided for region $\mathrm{AI}\left(\Delta_{1}=35.9^{\circ}\right)$, region AII $\left(\Delta_{1}=13.8^{\circ}\right)$ and region AIII $\left(\Delta_{1}=5.6^{\circ}\right)$. 
same width), which is reasonable in this experiment. A value of $\Delta_{i}=1.325 \theta_{i}$ is obtained for a perfectly Gaussian distribution, and a value of $\Delta_{i}=2 \theta_{i}$ is obtained for a perfectly bimodal distribution (no superposition). The values of $\Delta_{1}$ correspond to $2 \theta_{1}, 1.4 \theta_{1}$ and $1.3 \theta_{1}$ for AI, AII and AIII, respectively.

\section{References}

[1] J. Humphreys, G. Rohrer, A. Rollett, Recrystallization and Related Annealing Phenomena, Elsevier, 2017.

[2] B. Duggan, The problem of lost evidence, in: Term Discussed at the 11th ICOTOM Conference, Xian, China, 1996.

[3] D. Juul Jensen, H. Poulsen, The three dimensional X-ray diffraction technique, Mater. Charac. 72 (2012) 1-7.

[4] B. Larson, W. Yang, G. Ice, J. Budai, J. Tischler, Three-dimensional X-ray structural microscopy with submicrometer resolution, Nature 415 (2002) 887-890.

[5] D. Juul Jensen, Y. Zhang, Impact of 3D/4D methods on the understanding of recrystallization, Current Opinion in Solid State \& Materials Science (2020) 100821

[6] C. Xu, Y. Zhang, A. Godfrey, G. Wu, W. Liu, D. Juul Jensen, Direct observation of nucleation in the bulk of an opaque sample, Sci. Rep. doi: 10.1038/srep42508 (2017).

[7] S. West, S. Schmidt, H. Sørensen, G. Winther, H. Poulsen, L. Margulies, C. Gundlach, D. Juul Jensen, Direct non-destructive observation of bulk nucleation in 30\% deformed aluminum., Scripta Mater. 61 (2009) 875-878.

[8] M. Miszczyk, H. Paul, J. Driver, C. Maurice, New orientation formation and growth during primary recrystallization in stable single crystals of three facecentred cubic metals, Acta Mater. 83 (2015) 120-136.

[9] G. Wu, D. Juul Jensen, Orientations of recrystallization nuclei developed in columnar-grained $\mathrm{Ni}$ at triple junctions and a high-angle grain boundary, Acta Mater. 55 (2007). 4955-1964

[10] A. Godfrey, W. Cao, N. Hansen, Q. Liu, Stored Energy, Microstructure, and Flow Stress of Deformed Metals, Met. Mat. Trans, A 36 (2005) 2371-2378.

[11] G. Fan, Y. Zhang, J. Driver, D. Juul Jensen, Oriented growth during recrystallization revisited in three dimensions, Scr. Mater. 72-73 (2014) 9-12.

[12] V. Randle, N. Hansen, D. Juul Jensen, The deformation behaviour of grain boundary regions polycrystalline aluminium, Philos. Mag. 73 (1996) 265-282.

[13] R. Quey, P. Dawson, J. Driver, Grain orientation fragmentation in hot-deformed aluminium: Experiment and simulation, J. Mech. Phys. Solids 60 (2012) 509-524.
[14] R. Quey, J. Driver, P. Dawson, Intra-grain orientation distributions in hot-deformed aluminium: Orientation dependence and relation to deformation mechanisms, J. Mech. Phys. Solids 84 (2015) 506-527.

[15] J.-C. Glez, J. Driver, Orientation distribution analysis in deformed grains, J. Appl. Crystallogr. 34 (2001) 280-288.

[16] N.R. Barton, P.R. Dawson, On the spatial arrangement of lattice orientations in hot-rolled multiphase titanium, Model. Simul. Mater. Sci. Eng. 9 (2001) 433-463.

[17] W. Pantleon, W. He, T. Johansson, C. Gundlach, Orientation Inhomogeneities within Individual Grains in Cold-rolled Aluminium resolved by Electron Backscatter Diffraction, Mater. Sc. Eng., A 483-484 (2008) 668-671.

[18] A. Morawiec, Orientations and Rotations, Springer, 2004.

[19] R. Quey, A. Villlani, C. Maurice, Nealry uniform sampling of crystal orientations, J Appl Crys 51 (2018) 1162-1173.

[20] W. Read, W. Shockley, Dislocation Models of Crystal Grain Boundaries, Phys. Rev. 78 (1950) 275-289.

[21] L. Von, E. Murr, Interfacial Phenomena in Metal and Alloys, Addison-Wesley Publishing Company, 1975

[22] C. Barlow, B. Bay, N. Hansen, A comparative investigation of surface relief structures and dislocation microstructures in cold-rolled aluminium, Philos. Mag. A 51 (1985) 253-275.

[23] V. Randle, N. Hansen, D. Juul Jensen, The deformation behaviour of grain boundary regions in polycrystalline aluminium, Philos. Mag. A 73 (1996) 265-282.

[24] T. Ruggles, T. Rampton, A. Khosravani, D. Fullwood, The effect of length scale on the determination of geometrically necessary dislocations via EBSD continuum dislocation microscopy, Ultramicroscopy 164 (2016) 1-10.

[25] W. Pantleon, Unbiased Evaluation of Chord Length Distributions from Orientation Maps, Mater. Sci. Forum 495-497 (2005) 219-224.

[26] C. Xu, Y. Zhang, F. Lin, G. Wu, Q. Liu, D. Juul Jensen, Crystallographic Analysis of Nucleation at Hardness Indentations in High-Purity Aluminum, Metall Trans A 12 (2016) 5863-5870.

[27] Z. Zhang, Y. Zhang, O. Mishin, N. Tao, W. Pantleon, D. Juul Jensen, Microstructural Analysis of Orientation-Dependent Recovery and Recrystallization in a Modified 9Cr-1Mo Steel Deformed by Compression at a High Strain Rate, Metall Trans A 47 (2016) 4682-4693.

[28] J. Driver, The limitations of continuous dynamic recrystallization (CDRX) of aluminium alloys, Mater. Letters 2222 (2018) 135-137.

[29] M. Zecevic, R. Lebensohn, R. McCabe, M. Knezevic, Modelling recrystallization textures driven by intragranular fluctuations implemented in the viscoplastic self-consistent formulation, Acta Mater. 164 (2019) 530-546. 